\title{
Stator reference frame approach for DC injection-based stator resistance estimation in electric drives
}

\author{
Giovanni Zanuso*, Luca Peretti*, Paul Sandulescu* \\ *ABB Corporate Research, Department of Electrical Systems \\ Forskargränd 7, 72178 Västerås, Sweden \\ Email: giovanni.zanuso@se.abb.com, luca.peretti@se.abb.com, paul.sandulescu@se.abb.com
}

\begin{abstract}
This work discusses an online stator resistance estimation method for synchronous machines. Multiple improvements are provided with respect to a similar method already been used for induction machines. The method is based on the temporary injection of a DC voltage in the machine phases during normal operation. The stator resistance estimation is obtained by means of the Ohm's law. The proposed scheme works entirely in the stator fixed reference frame, without the need of any machine parameter.

The selection of the DC injection level is discussed, as well as the countermeasures for reducing the spurious harmonics appearing in the currents and the torque when the machine is speed- and current-regulated. In particular, minimum electric loss and minimum torque ripple due to the injection is achieved. Moreover, as an alternative to conventional low-pass filtering of the measured signals, it is shown that the resistance estimation can be obtained by averaging the signals over few electric periods. Experiments prove the validity of the proposed approach.
\end{abstract}

\section{INTRODUCTION}

In recent years, an increasing number of electrical drives research activities has been devoted to the on-line estimation of the stator resistance in electrical machines by means of DC voltage injection methods ([1], [2], [3], [4], [5], [6]). Primarily driven by the need to estimate the temperature variation of the machine by indirectly tracking the variation of stator resistance ([3], [6]), these methods can potentially be used even to provide a reliable resistance value for model-based control algorithms, including speed-sensorless algorithms. This is a consequence of the nature of these techniques, which rely on the injection of DC voltage signals and the analysis of the induced DC currents. Thus, no information on the electrical machine is required, avoiding schemes based on models that require the knowledge of inductances.
Among the known methodologies, it is common to use an oscillatory torque injection over the torque reference applied in a direct torque control (DTC) scheme [3] or a DC flux injection over the flux reference, again in a DTC scheme [4]. Another alternative regards the injection of DC voltages performed in the fixed stator reference frame (either $\alpha \beta$ or $a b c$ ) [6].

In both cases, the typical three-phase voltages and currents of the machine present a non-zero DC value for a short time interval during drive operation. By means of the Ohm's law, the value of the resistance is obtained.

One of the main drawbacks of the injection-based resistance estimation methods is that the DC levels in the $\alpha \beta$ or $a b c$ reference frame are disturbances in the rotating $d q$ reference frames, causing the introduction of torque harmonics and disturbing the normal operation of the drive. It has been shown for some application cases that these harmonics are sufficiently small not to cause any concern [3].

Another drawback of some injection-based solutions relates to the injection location in the control chain. If this is performed in the rotating reference frame, a non-ideal situation occurs when the control algorithm is running in speed-sensorless mode. In order to estimate the speed and the position of the reference frame, the estimation of the flux linkage is required. When the latter is obtained as the integration of the back-electromotive force, the stator resistance value is needed. Thus, the injection methods performed in the $d q$ reference frame could be potentially dependent on the value of the stator resistance itself. In order to avoid any trouble between causes and effects, the estimation scheme should be an independent entity from the control scheme. For this reason, solutions running in the $\alpha \beta$ or $a b c$ reference frames as [6] should be preferable.

The present work further investigates the findings of 
[6], by discussing a slightly different estimation scheme applied to a synchronous reluctance machine (SynRM), and by successively proposing some enhancements to reach minimum harmonic interaction with the control and minimum electrical losses caused by the DC voltage injection. The fundamental equations are shown in Sect. II-A, while the basic estimation scheme is presented in Sect. II-B. Sect. II-C discusses an alternative way to calculate the DC currents which is not based on lowpass filters, while Sect. II-D presents the modifications required to obtain minimum DC current injection and minimum harmonic interaction with the control. Experimental tests are reported in Sect. III, followed by validation cases in Sect. IV where the estimation has been compared to a four-wire multimeter measurement of the stator resistance.

\section{THEORETICAL BACKGROUND}

\section{A. Fundamental equations}

The space-vector equation describing the dynamics of a SynRM in a stator-fixed $\alpha \beta$ reference frame is the following:

$$
\boldsymbol{u}_{\alpha \beta}=R_{s} \boldsymbol{i}_{\alpha \beta}+\frac{d \boldsymbol{\lambda}_{\alpha \beta}}{d t}
$$

where $\boldsymbol{u}_{\alpha \beta}, \boldsymbol{i}_{\alpha \beta}$ and $\boldsymbol{\lambda}_{\alpha \beta}$ are the space vectors of the stator voltage, the stator current and the stator flux linkage respectively, and $R_{s}$ is the stator resistance. A description in the $d q$ reference frame, rotating with the electro-mechanical rotor angle $\vartheta_{m e}$, is given below:

$$
\boldsymbol{u}_{d q}=R_{s} \boldsymbol{i}_{d q}+\frac{d \boldsymbol{\lambda}_{d q}}{d t}+j \omega_{m e} \boldsymbol{\lambda}_{d q}
$$

where $\omega_{m e}$ is the electro-mechanical rotor speed, and $\boldsymbol{u}_{d q}, \boldsymbol{i}_{d q}$ and $\boldsymbol{\lambda}_{d q}$ are the $d q$ space vectors of the stator voltages, the stator currents and the stator flux linkages respectively.

In presence of a field-oriented control (FOC) scheme, the closed-loop regulation tends to cancel the crosscoupling term in (2). Thus, assuming an ideal closedloop current control, the expression (2) becomes:

$$
\boldsymbol{u}_{d q}=R_{s} \boldsymbol{i}_{d q}+\frac{d \boldsymbol{\lambda}_{d q}}{d t}
$$

If (3) is transformed back into the stator-fixed reference frame, it gives:

$$
\boldsymbol{u}_{\alpha \beta}=R_{s} \boldsymbol{i}_{\alpha \beta}+\frac{d \boldsymbol{\lambda}_{\alpha \beta}}{d t}-j \omega_{m e} \boldsymbol{\lambda}_{\alpha \beta}
$$

This equation is very important for its effects on the selection of the DC voltage injection.

\section{B. Basic estimation scheme}

A FOC for a SynRM merged with the basic stator resistance estimation scheme is shown in Fig. 1, where SVM indicates a conventional space vector modulation.

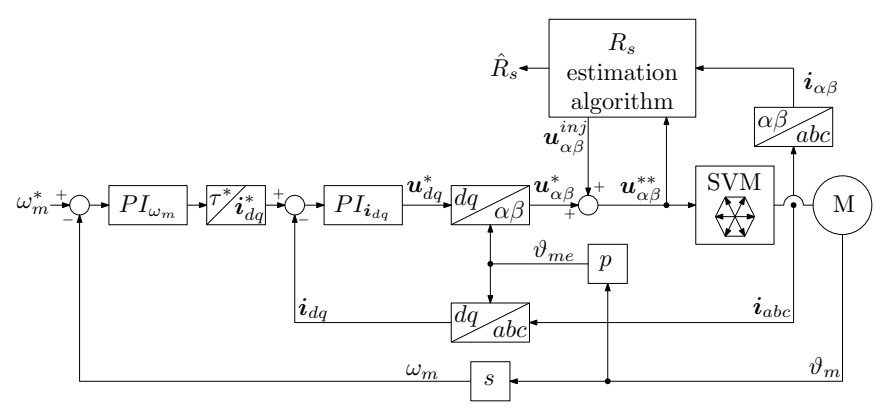

Fig. 1. FOC diagram with the basic resistance estimation.

The estimation algorithm operates in the $\alpha \beta$ reference frame, by applying an injection signal $\boldsymbol{u}_{\alpha \beta}^{i n j}$ of the form:

$$
\boldsymbol{u}_{\alpha \beta}^{i n j}=u_{\alpha, D C}^{i n j}+j 0
$$

where $u_{\alpha, D C}^{i n j}$ is a DC value. Provided that a proper compensation for the IGBT dead times and the SVM delay is performed, the stator resistance estimation is obtained as:

$$
\hat{R}_{s}=\frac{u_{\alpha, D C}^{* *}}{i_{\alpha, D C}}
$$

where $u_{\alpha, D C}^{* *}$ and $i_{\alpha, D C}$ are the DC values of the $\alpha$-axis components of the vectors $\boldsymbol{u}_{\alpha \beta}^{* *}$ and $\boldsymbol{i}_{\alpha \beta}$, respectively (see Fig. 1). Two aspects are worth noting:

- Due to (4), when $\omega_{m e} \neq 0$, a DC current is not only induced on the $\alpha$ axis, but on the $\beta$ axis as well. The presence of an offset in $i_{\beta}$ is considered a drawback as it will be explained in Sect. II-D.

- Since the current DC values are seen as disturbances by the FOC scheme, the latter tries to reject them by generating voltage harmonics multiple of the rotating frequency in the $d q$ frame, which are translated into DC voltages in the $\alpha \beta$ frame. The FOC scheme employs PI regulators, therefore no complete rejection is achieved and spurious current DC offsets can be observed in $\alpha \beta$. As an overall result, this leads to $u_{\alpha, D C}^{* *} \neq u_{\alpha, D C}^{i n j}$.

An accurate estimation of the stator resistance is obtained when the DC component of $\boldsymbol{i}_{\alpha \beta}$ is large enough to avoid sensor resolution issues. However, the DCoffset rejecting capabilities of the FOC are difficult to predict especially in presence of non-linear magnetic circuits as in the SynRM, and therefore no a-priori $u_{\alpha, D C}^{i n j}$ selection can be made. As a consequence, $u_{\alpha, D C}^{i n j}$ must 
be the result of a closed-loop regulation, as proposed in [6] and as shown in Fig. 2. $u_{\alpha, D C}^{i n j}$ is the output of a PI regulator which controls $i_{\alpha, D C}$ to a reference value $i_{\alpha, r e f}$, the latter being set to guarantee a sufficiently small quantisation error on the measured phase currents.

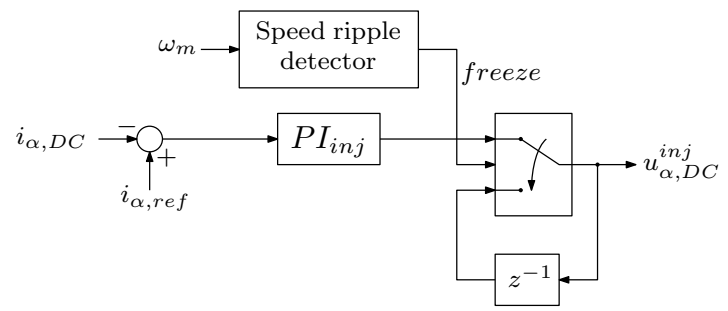

Fig. 2. $u_{\alpha, D C}^{i n j}$ generation with speed ripple limitation.

Fig. 2 shows also that the amplitude of $u_{\alpha, D C}^{i n j}$ depends on the speed ripple detection block. When the speed ripple with respect to the speed average value exceeds a limit $\widetilde{\omega}_{m, \max }, u_{\alpha, D C}^{i n j}$ is maintained constant through the activation of the signal freeze. This additional feature was included to avoid the drive to generate excessive speed oscillations as a result of the torque harmonics generated by the DC voltage injection.

\section{Alternative offset calculation}

The DC values required by (6) could be calculated by using low-pass filters. Their presence require a tuning of their cut-off frequency, possibly implying a slower convergence of the estimated value $\hat{R}_{s}$. Alternatively, the signals $\boldsymbol{u}_{\alpha \beta}^{* *}$ and $\boldsymbol{i}_{\alpha \beta}$ are known to contain harmonics multiple of the electrical rotor frequency $f_{m e}$. Therefore, their DC values could be extracted by calculating their average over an electrical period, automatically eliminating the effect of the harmonics.

A drawback of this method is that the electrical period $T_{m e}=1 / f_{m e}$ is not always an exact multiple of the current sample time $T_{c}$. Should this happen, the obtained average value is inaccurate and exhibits an oscillatory behaviour. However, if the signal is computed over a certain number of electrical periods $N$, with $N T_{m e}$ being an exact multiple of $T_{c}$, the result will be equal to its avergae value. Therefore, $N$ must satisfy the following condition:

$$
N: N \cdot \gamma \in \mathbb{N}
$$

where $\gamma$ is the fractional part of the division between $T_{m e}$ and $T_{c}$, as calculated below:

$$
\gamma=\left\{\frac{T_{m e}}{T_{c}}\right\} \triangleq \frac{T_{m e}}{T_{c}}-\left\lfloor\frac{T_{m e}}{T_{c}}\right\rfloor
$$

The pure application of the condition (7) leads inevitably to a very large value of $N$ and, as a consequence, to a very long computation time $N T_{m e}$. An alternative is to consider only a number $N^{\prime}$ which ensures a processing time below a limit $T_{\text {lim }}$ while minimising the fractional part $\left\lfloor N^{\prime} \gamma\right\rfloor$ :

$$
N^{\prime}:\left\{\begin{aligned}
\left\lfloor N^{\prime} \gamma\right\rfloor & =\min (\lfloor N \gamma\rfloor) \\
N^{\prime} T_{m e} & <T_{\text {lim }}
\end{aligned}\right.
$$

The choice of the parameter $T_{l i m}$ is a compromise between the estimation execution time and its accuracy.

\section{Improved estimation scheme}

In Sect. II-B, it was mentioned that a DC voltage injection in the $\alpha$ axis does not induce only a DC current in the $\alpha$ axis. Moreover, if no further action is taken, the presence of the DC injection is considered as a disturbance by the FOC algorithm, which tries to reject it and contributes to an increase of the time execution of the stator resistance algorithm.

A way to deal with this problem is described in [6], where the DC value of the current $i_{\alpha}$ is subtracted from the measured $i_{\alpha}$ that is used for the FOC feedback loop. In this way, the current PI regulators in the $d q$ reference frame do not receive any information on the DC injection, thus not attempting to reject them.

Although successful for the purpose of decreasing the estimation execution time, this method does not minimise completely the current injection magnitude for a given desired value of DC offset on $i_{\alpha}$. This is again due to (4): a voltage injection on the $\alpha$ axis generates a non-zero offset value of $i_{\beta}$ too.

The presence of an offset on $i_{\beta}$ is considered as a drawback, since it increases the torque ripple and the copper losses during the estimation without any benefit for the algorithm, which is only using the $i_{\alpha, D C}$ value to perform the estimation in the original basic scheme of Sect. II-B. Furthermore, the DC offset on $i_{\beta}$ is fed back to the PI regulators, which will react to that and slow down the execution of the algorithm.

The solution proposed in this work is to control to zero $i_{\beta, D C}$ by adding another regulation loop as shown in Fig. 3. In this way, the injected voltage assumes the following form:

$$
\boldsymbol{u}_{\alpha \beta}^{i n j}=u_{\alpha, D C}^{i n j}+j u_{\beta, D C}^{i n j} .
$$

Moreover, similarly to what is proposed in [6] for $i_{\alpha}$, the offset on $i_{\beta}$ is also removed for the signals that are fed back to the PI regulators. In this way the current/torque loop will not interact with the estimation 
algorithm. The amended proposed estimation scheme combined with the FOC is shown in Fig. 4.

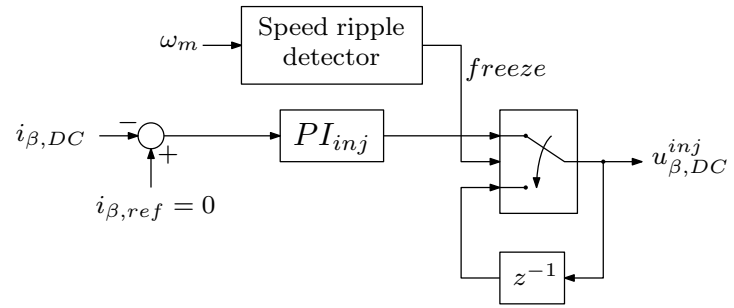

Fig. 3. $u_{\beta, D C}^{i n j}$ generation with speed ripple limitation.

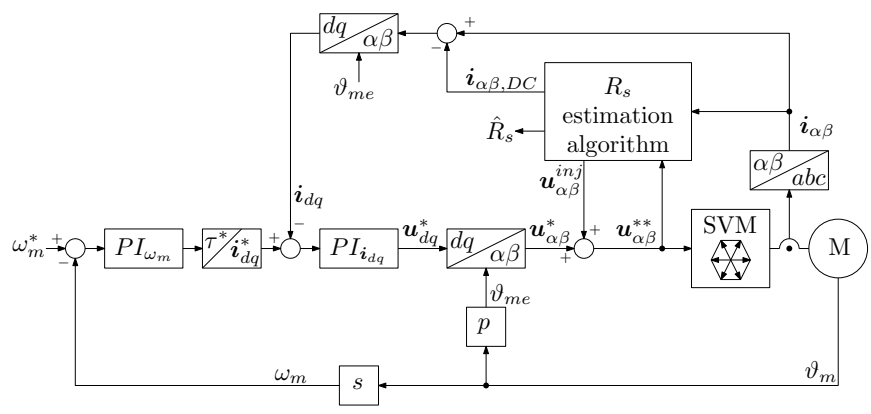

Fig. 4. Conventional FOC diagram with proposed resistance estimation.

\section{EXPERIMENTAL RESULTS}

\section{A. The experimental setup}

The proposed solution was implemented on an OPALRT OP5600 control system, equipped with a quad-core Intel DSP processor at $2.4 \mathrm{GHz}$ and a Virtex 6 FPGA. The system was connected to the power unit of an $\mathrm{ABB}$ ACS850 converter through a custom interface board. An 11-kW SynRM, whose parameters are reported in Tab. II in the Appendix, was driven by the converter. A 11$\mathrm{kW}$ Baldor machine, controlled by an off-the-shelf $\mathrm{ABB}$ ACS850 converter, was connected as the load machine.

\section{B. Test of the basic estimation scheme}

Fig. 5 shows the behaviour of the algorithm described in Section II-B, when the rotating speed was controlled at $\omega_{m}^{*}=50 \mathrm{rad} / \mathrm{s}$ and no load torque was applied. The parameters used in the estimation algorithm are listed in Tab. III. At approximately $2 \mathrm{~s}$, the resistance estimation algorithm is activated and thus a DC voltage is injected only in the $\alpha$ axis. Since in this first version the voltage $u_{\beta, D C}^{i n j}$ is not controlled, an offset appears on the $\beta$ axis current $\left(i_{\beta, D C} \neq 0\right)$. The DC injection increases the amount of ripple in $i_{d q}$, as visible in Fig. 5, while the speed ripple is not significantly affected due to the inherent filtering action of the mechanical system. In order to avoid any inaccurate result of $\hat{R}_{s}$, the operation (6) is prompted only when $i_{\alpha, D C}$ is within the interval $\left[i_{\alpha, r e f}-\epsilon_{i}, i_{\alpha, r e f}+\epsilon_{i}\right]$. Thus, the first value of $\hat{R}_{s}$ is available around $20 \mathrm{~s}$ after the algorithm activation.
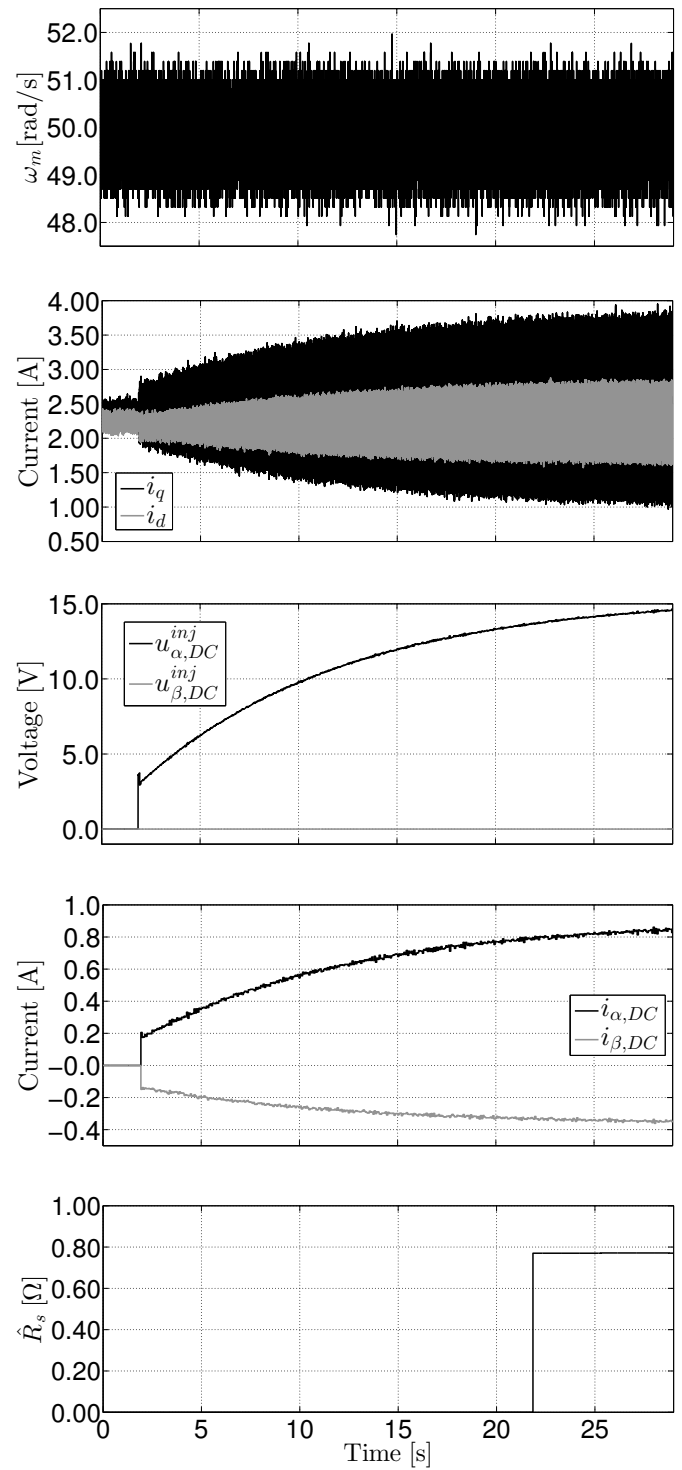

Fig. 5. $\hat{R}_{s}$ with basic estimation scheme for $\omega_{m}^{*}=50 \mathrm{rad} / \mathrm{s}$.

\section{Test of the improved estimation scheme}

Fig. 6 reports the behaviour of the algorithm described in Section II-D, where the voltage injection is controlled on both $\alpha$ and $\beta$ axes, and the current offset vector $\boldsymbol{i}_{\alpha \beta, D C}$ is subtracted from the current vector $\boldsymbol{i}_{\alpha \beta}$, which is fed back to the $d q$ current regulation. The speed reference, the load torque condition and the estimator parameters are the same as the ones used in the test of Sect. III-B. 
The removal of $\boldsymbol{i}_{\alpha \beta, D C}$ from the measured current leads to some differences with respect to the results of the previous test. The ripple on the currents $i_{d}$ and $i_{q}$ is obviously reduced, which involves also a reduction of the PI regulators rejection activity. As a consequence, the magnitude of the injected voltage vector $\boldsymbol{u}_{\alpha \beta}^{i n j}$ is smaller. The estimation execution time is also reduced, with the first value of $\hat{R}_{s}$ available around $4 \mathrm{~s}$ after the algorithm activation.

Since the offset current $i_{\alpha, D C}$ is controlled to the same $i_{\alpha, r e f}$ value as in the previous test, and the $i_{\beta, D C}$ is instead controlled to zero, the magnitude of the induced DC current vector $\boldsymbol{i}_{\alpha \beta, D C}$ is the smallest possible. Therefore, electric losses and torque ripple are also reduced at minimum.
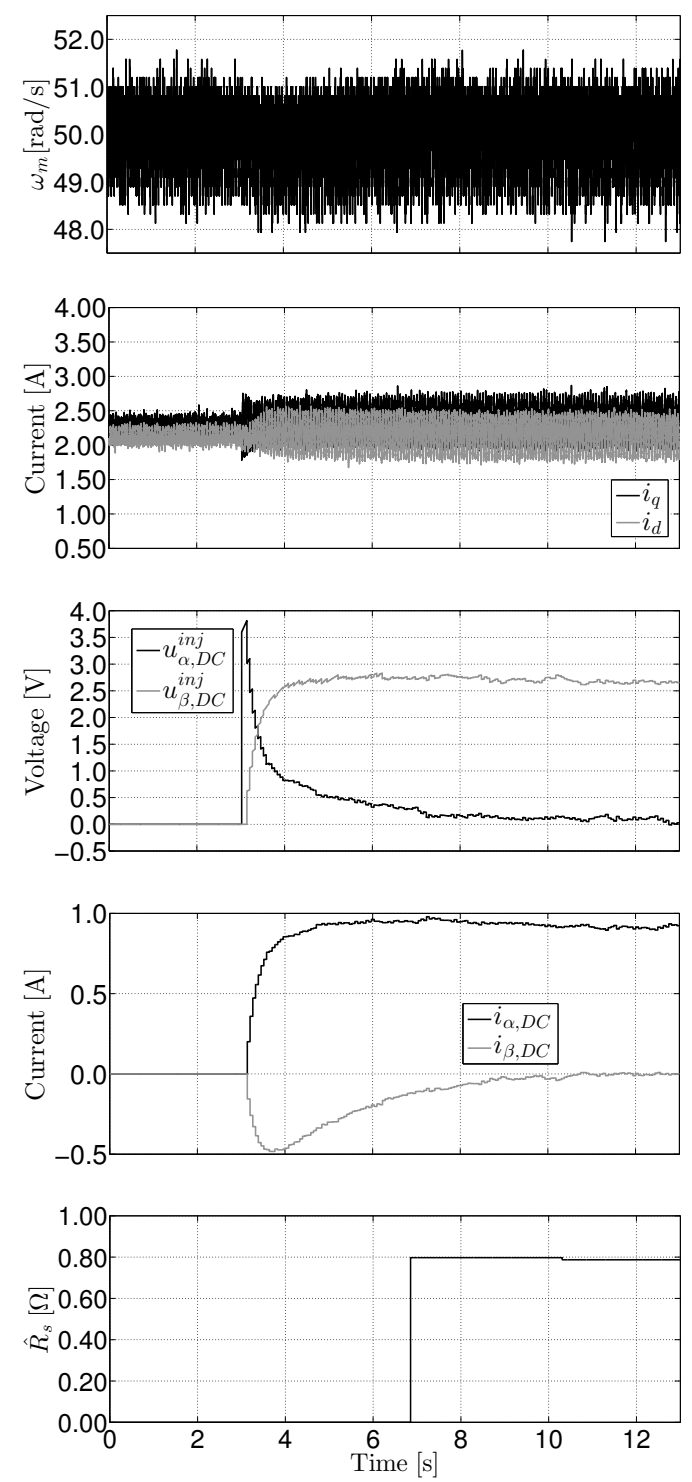

Fig. 6. $\hat{R}_{s}$ with improved estimation scheme for $\omega_{m}^{*}=50 \mathrm{rad} / \mathrm{s}$.

\section{VALIDATION}

The values of $\hat{R}_{s}$ were compared to four-wire resistance measurements performed by a Hewlett Packard 34401A multimeter.

It is worth noting the slight difference between the estimation performed by the algorithm and that obtained by the measurements. The estimation algorithm is based on the Ohm's law between the voltage references and the stator current measurements. Therefore, the estimated stator resistance $\hat{R}_{s}$ includes the resistance of the machine windings, the resistance of the cable connecting the converter to the machine and the resistive component of the switches in the converter. On the other hand, since the four-wire measurement is applied at the output terminals of the converter, it will not include the resistive contribution of the components inside the converter.

Another limit of the proposed validation method is the difficulty to measure the stator resistance with the multimeter while the current is flowing. Thus, the fourwire measurement must be executed when the drive is shut down and the converter switches are open.

Considering the aforementioned limitations, the validation test has been carried out five times in order to reduce random errors. The procedure for the validation of the estimation algorithm consists on the following steps:

1) With the drive turned off and the cold SynRM, the four-wire resistance measurement is executed between all phase terminal pairs of the converter $a b, b c$ and $a c$. The three phase-to-phase resistances $R_{a b}, R_{b c}$ and $R_{a c}$ are averaged and divided by two to get an estimation of the stator resistance $R_{s}$ :

$$
R_{s}=\frac{R_{a b}+R_{b c}+R_{a c}}{6}
$$

2) The drive is turned on and the resistance estimation algorithm is activated as fast as possible. The estimation is performed with a speed of $\omega_{m}^{*}=$ $5 \mathrm{rad} / \mathrm{s}$, set by the FOC speed regulation, with no load torque. In these conditions, the phase currents are not large enough to significantly alter the stator resistance value due to the temperature variation.

3) The machine is warmed-up for a while, in order to obtain a larger stator resistance value. The reference speed is set to $\omega_{m}^{*}=50 \mathrm{rad} / \mathrm{s}$ with a load torque of $\tau_{L}=11.88 \mathrm{Nm}$. The estimation algorithm output $\hat{R}_{s}$ represents the "warm" resistance case.

4) The drive is immediately turned off and the phaseto-phase four-wire measurements with the multi- 
meter are performed, as in the first step of the procedure.

Tab. I reports the validation results for the cold resistance case (steps 1 and 2) and the warm resistance case (steps 3 and 4). Both cases show that $\hat{R}_{s}$ is smaller than $R_{s}$ and that the maximum relative error is in the order of $3 \%$. Different causes could be considered for this systematic offset (imperfect dead-time compensation, current sensors accuracy, etc.), but the overall accuracy of the method is good and robust.

It is worth noting that both tables have five rows of results, each one corresponding to one test. A comparison between the results of different rows is meaningless, since the thermal condition of the drive between distinct tests is not the same.

Tab. I

VALIDATION OF THE STATOR RESISTANCE ESTIMATION.

\begin{tabular}{c|c|c|c}
\hline \multicolumn{4}{c}{ Cold resistance case } \\
\hline Test & $R_{s}[\Omega]$ & $\hat{R}_{s}[\Omega]$ & $\epsilon_{r \%}[\%]$ \\
\hline 1 & 0.7130 & 0.7070 & -0.84 \\
\hline 2 & 0.7248 & 0.7177 & -0.98 \\
\hline 3 & 0.7120 & 0.7085 & -0.49 \\
\hline 4 & 0.7243 & 0.7025 & -3.00 \\
\hline 5 & 0.7208 & 0.7000 & -2.89 \\
\hline \multicolumn{4}{|c}{ Warm resistance case } \\
\hline Test & $R_{s}[\Omega]$ & $\hat{R}_{s}[\Omega]$ & $\epsilon_{r} \%[\%]$ \\
\hline 1 & 0.7517 & 0.7510 & -0.09 \\
\hline 2 & 0.7633 & 0.7570 & -0.83 \\
\hline 3 & 0.7683 & 0.7565 & -1.54 \\
\hline 4 & 0.7595 & 0.7425 & -2.24 \\
\hline 5 & 0.7833 & 0.7730 & -1.32 \\
\hline
\end{tabular}

\section{CONCLUSIONS}

An online stator resistance estimation method by means of a DC voltage injection in the stator reference frame of a SynRM was analysed. The method is suitable for resistance estimation without any knowledge of the machine parameters. The effects of the DC voltage injection on the FOC were discussed, and countermeasures were taken in order to minimise the induced DC current, the torque ripple and the algorithm execution time. In particular, although only the induced current on the $\alpha$ axis is considered for the resistance estimation, a regulation scheme for both the $\alpha$ and $\beta$ axis is proposed, as this is a possible way to obtain the minimum electrical losses and torque ripple. Moreover, as an alternative to conventional low-pass filtering for extracting the DC value of the signals, the current and voltage DC offsets are obtained by an average over multiple electric periods.
The experiments and the validation of the estimation algorithm have shown relative errors in the range up to $3 \%$ for the case under investigation, proving the effectiveness of the method.

\section{REFERENCES}

[1] S.-B. Lee and T. G. Habetler, "An online stator winding resistance estimation technique for temperature monitoring of lineconnected induction machines," IEEE Trans. Ind. Appl., vol. 39, no. 3, pp. 685-694, May/Jun. 2003.

[2] — "A remote and sensorless thermal protection scheme for small line-connected AC machines," IEEE Trans. Ind. Appl., vol. 39, no. 5, pp. 1323-1332, Sep./Oct. 2003.

[3] L. He, S. Cheng, R. G. Harley, and T. G. Habetler, "A torqueinjection-based approach for thermal monitoring of induction machines with direct torque control," in Proceedings of the International Electrical Machines and Drives Conference (IEMDC), Chicago, Illinois, USA, May 12-15, 2013, pp. 93-99.

[4] L. He, S. Cheng, Y. Du, R. G. Harley, and T. G. Habetler, "A DC-flux-injection approach for thermal monitoring of induction machines with direct torque control," in Proceedings of the Energy Conversion Congress and Exposition (ECCE), Denver, Colorado, USA, Sep. 15-19, 2013, pp. 2115-2122.

[5] _ - "Stator temperature estimation of direct-torque-controlled induction machines via active flux or torque injection," IEEE Trans. Power Electron., 2014, early access article.

[6] P. Matic, M. Gecic, D. Lekic, and D. Marcetic, "Thermal protection of vector-controlled im drive based on dc current injection," Industrial Electronics, IEEE Transactions on, vol. 62, no. 4, pp. 2082-2089, April 2015.

\section{APPENDIX}

Tab. II

11-KW SYNRM PARAMETERS.

\begin{tabular}{cc}
\hline Parameter & Value \\
\hline$R_{s}$ & $0.72 \Omega$ \\
$L_{d}$ (non-saturated value) & $80 \mathrm{mH}$ \\
$L_{q}$ (non-saturated value) & $60 \mathrm{mH}$ \\
$p$ & 2 \\
$u_{n}$ & $400 \mathrm{~V}$ \\
$i_{n}$ & $18 \mathrm{~A}$ \\
$\omega_{n}$ & $647.05 \mathrm{rad} / \mathrm{s}$ \\
$\tau_{n}$ & $17 \mathrm{Nm}$ \\
$J_{m}$ & $105 \cdot 10^{-3} \mathrm{~kg} \mathrm{~m}{ }^{2}$ \\
$B_{m}$ & $10 \cdot 10^{-3} \mathrm{Nm} \mathrm{s} / \mathrm{rad}$ \\
\hline
\end{tabular}

Tab. III

RESISTANCE ESTIMATOR PARAMETERS.

\begin{tabular}{cc}
\hline Parameter & Value \\
\hline$i_{\alpha, \text { ref }}$ & $0.9 \mathrm{~A}$ \\
$K_{p, \text { inj }}$ & $4 \mathrm{~V} / \mathrm{A}$ \\
$K_{i, \text { inj }}$ & $2 \mathrm{~V} /(\mathrm{As})$ \\
$\widetilde{\omega}_{m, \max }$ & $5 \mathrm{rad} / \mathrm{s}$ \\
$T_{\text {lim }}$ & $5 \mathrm{~s}$ \\
$\epsilon_{i}$ & $0.1 \mathrm{~A}$ \\
\hline
\end{tabular}

